\title{
Sciendo
}

ACTA UNIVERSITATIS CIBINIENSIS - TECHNICAL SERIES

DOI: $10.2478 /$ aucts-2019-0015

Vol. 712019

\section{MAIN REQUIREMENTS OF A CYBER PHYSICAL PRODUCTION SYSTEM DEMONSTRATOR}

\author{
PETRUSE Radu Emanuil \\ Faculty of Engineering, Department of Industrial Engineering and Management, "Lucian Blaga" \\ University, Sibiu, Romania, radu.petruse@ulbsibiu.ro \\ BONDREA Ioan \\ Faculty of Engineering, Department of Industrial Engineering and Management, "Lucian Blaga" \\ University, Sibiu, Romania, ioan.bondrea@ulbsibiu.ro \\ NICOLAE Ioan Cristian \\ Faculty of Engineering, Department of Industrial Engineering and Management, "Lucian Blaga" \\ University, Sibiu, Romania, ioancristiannicolae@yahoo.com
}

\begin{abstract}
The paper is focused on identifying the main requirements for developing a CPPS demonstrator prototype. This research aims to categorize the main components and interactions within a new manufacturing system which are required to accommodate a CPPS. The proposed CPPS demonstrator also includes the preliminary requirements identified from the discussions with the regional industry, so that the prototype of the production system could be easily understood and also be the basis for further collaborations. The identified requirements were structured according to the top-down principle focusing on accomplishing the major objectives to be achieved through the prototype of the production system but also on the detailed concepts of the product and the production stations, thus preparing the choice for a concrete technical solution.
\end{abstract}

Key words: Industry 4.0, Cyber-Physical Systems

\section{Introduction}

Since the first industrial revolution there has been a constant struggle to optimise the production processes, obtain better manufacturing times, increase the quality of the products and satisfy the customers' demands. The third industrial revolution brought with it the rise of electronics and of the programmable machines. The two major inventions that marked that period were the PLC and the robots.

Nowadays machines have been upgraded to the point that they became indispensable in a company due to their impact on the efficiency of the processes and lately because of the "intelligent" way of solving problems. Modern machines and industrial equipment can be perceived similar to an intelligent entity which has senses like sight, hearing, touch, taste and smell, is able move in the physical environment and most important it thinks before taking action.

At first robots were used strictly for actuation (to mimic the movements the human body does). The first major progress towards a smart productions system was made when the industrial equipment (e.g. robots, CNC machines, automation devices, etc.) were upgraded with sensors which made them aware of their surroundings (e.g. proximity sensors, temperature sensors etc.) and the processes that are developing around them. The only missing part towards a smart productions system was the cognition process. Industry 4.0 is trying to solve this problem, by connecting each computing device, mechanical and digital machine, robot and other significant equipment, to a system called Internet of Things (IoT). With the help of IoT, information about the state of the machines, the tools that are in use and about the products, is being sent in real time through a communication network. The engineer or the technician is be able to act promptly if anything seems to work incorrectly or if the process is not running at an optimum. By taking fast action in order to solve the problem, the costs on the long term of the companies will drop and the quality of their products will rise. Also, a simulation of a different process can be performed with live data from production system, thus enabling a dynamic production planning [1]. With all these, IoT has some disadvantages as well. For example, it is hard to secure such a huge amount of information that is being sent inside the 
company generating a big concern regarding the cybersecurity, intellectual property and the data privacy which could be at risk if the network is hacked.

Industry 4.0 describes an organisation in which the processes take place autonomously due to the communication between every machine and robot. In this type of organization computer-driven systems monitor physical processes, develops a digital twin of the physical world and is able to make decentralised decisions based on self-organisation mechanisms (e.g. agents) in the smart factory. By making the robots and machines aware of their environment, they will be able to "negotiate" their tasks thus improving the whole production process. [1] [2]

A Cyber Physical System (CPS) is a mechanism that is controlled and/or monitored with the help of algorithms via a remote connection which is able to communicate, adapt and learn, with the help of artificial intelligence. Advanced companies are trying to move towards a Cyber Physical Production System (CPPS) which is able to meet the expectations of more informed and demanding customers. A CPPS consists of CPS's that are connected peer to peer to each other this way they can access information gathered by each one of them and use it in order to optimize the production process. A peer-to-peer framework decentralizes the decision-making process, eliminating the possibility of having only one source of viable information. The connection of the physical and cyber world is relying on data acquisition from the physical world. Information gathered in the physical world will be transferred in the cyber world. The latter one is responsible with sending the right feedback to the CPS's in work, which will basically affect the results obtained with the end product existing in the physical world. CPPS are able to gain information on their own, without any input from the workers, using sensors and the information they gain from other CPS's. A CPPS is composed from modules which can be changed in order to get the desired result [4]. Each module will serve a different task: assembly, storage, manufacturing, etc.

The main design principles of a of CPPS are:

- Decision-making and execution decentralization (every machine will be able to take decisions on its own)

- Modularization of the production system and flexible reconfiguration of production control systems (dividing the production process into small steps that can be interchangeable, to help creating custom products without changing much to the assembly line)

- Adaptive connection of all production participants (every device in the factory should be connected to each other, this way they will deliver important information regarding the state of the production)

- Plug and Produce (it pleases expectation that an industrial networking or automation product can be simply connected and turned on - and it will work)

- Decentralized Production Planning System (there will not be a single source of information; every CPS will be its own information provider)

- Miniaturization of equipment and smaller production lines (optimization of factory space)

\section{Structure of the Cyber Physical Production System}

Taking into account the regional opportunities in conjunction with the general characteristics of the CPPS we identified the main objectives that the CPPS must achieve. The objectives were structured according to the top-down principle, as follows:

\subsection{General objectives}

Development of a research infrastructure suitable for the validation of the concepts of CPPS (Industry 4.0) implying that:

- The manufactured product has to have a high degree of customization;

- Each production module (workstation) should be equipped with sensory, computing, operating and communication capabilities that provide data, information and services to its local environment or to a cloud environment

- Each production module should be aware of the functionality it can offer (e.g. assembly capabilities) the current state (e.g. operational, defective, in working condition, etc.), what types of resources it needs for operation and operation (e.g. power supply, semi-manufactured, electrical components, etc.) but also the possibility to interact with other workstations within the manufacturing system (e.g. to negotiate the fastest way of producing the product)

\subsection{Specific objectives}

The CPPS must be able to fabricate a customizable product (through hardware and software adaptation) which allows: 
- A reconfiguration of the workstation topology / layout, depending on spatial and functional constraints;

- Adapting the resource (e.g. workstation, the product being manufactured) to failures through selfmanagement. For example, if a sensor no longer works, the system will find autonomous/independent alternatives to complete the order;

- Customization depending on the customer (e.g. if a customer gives up an order / adjusts the order during production, it is reused for another order);

- Price adjustment (resource consumption) depending on the quality of the product (e.g. a product with fewer finishing processes having a lower cost);

- Adaptation of the manufacturing process to changes in the product (e.g. the diameter of the housing changes, the order of operations for a new product will be different and the production devices must be adapted accordingly);

- The existence of redundant manufacturing stations or processes for product fabrication, which allow a wide spectrum of optimizations:

$\circ$ Time of product realization;

- Consumption of resources (energy, raw materials, etc.);

- Number of products made over a period of time;

- The degree of use of the equipment (e.g. machine tool to operate as long as possible);

- Just-in-time production.

2.3. Objectives for maximizing the impact for the regional industrial environment Incorporation of the following characteristics into the CPPS:

- Using OPC-UA, IO-Link and RFID standards for communication between stations;

- Highlighting the plug \& produce concept for easier reconfiguration of production modules/units based on the above standards;

- Highlighting the benefits of human-machine collaboration (e.g. collaborative robots) in an easily understandable industry scenario;

- Intelligent human assistance in production (e.g. assistance in carrying out tasks, assisting in commissioning equipment, etc.) in order to increase productivity.

\subsection{Objectives for maximizing the research impact}

The CPPS demonstrator has to be used for research activities that will focus on:

- Development of industrial agents for optimizing resources and processes such as:

- Simulation of interactions between industrial agents (e.g. components, type of interactions, content of messages, etc.);

- Semantic description of services.

- Servicing processes and products: providing services during production (e.g. real-time information on the ordering stage, offers during production - if someone has given up the order -) or after purchase (e.g. offering additional features)

- Digital Thread: development of approaches that contribute to the realization of a digital twin of the real manufacturing system, which will include semantics, graphical representation suitable for different engineering aspects (e.g. mechanics, electricity, control etc.) which also allows the co-simulation between the product and the manufacturing process.

\section{CPPS development}

The whole CPPS demonstrator concept is based on the product that it will be able to manufacture. This being the case, being a product-centric system, the fabricated product aims to fulfil two major characteristics:

- During its production to interact with the production system being able to store and provide information about its manufacturing processes;

- During the use (post-production) to offer a customizable functionality to the customer according to the different modules in the product's composition. For example, the product allows the use of mobile applications to perform various functions such as: location, monitoring, entertainment, etc.

The interaction between the customer, the production system and/or the product, aims to establish the desired product personalization. The customer's customization can represent the choosing, for example through a Web application, of different components for the finished product but also special features of the product (e.g. name engraving). Moreover, the customization can also imply the client's opportunity to choose the fabrication method for each product: a faster manufacturing (maximum performance mode), a 
manufacturing that involves a low energy consumption (eco mode) or a balanced manufacturing mode (Normal mode). Last but not least, the customer must receive feedback regarding the execution stage of the ordered product, being able to offer for example the possibility to customize/modify the product while it is still on the production line. The interaction between the product and the production system starts from the premise that the system received the customer's order regarding the product's particularization (e.g. two components, ECO production mode). An order management system takes the data entered by the client in the web application and mediates/collaborates with the production modules to complete the order. Through the web application, the customer can monitor in real time the evolution of the ordered product.

\subsection{Resulted CPPS characteristics}

Each CPS module is equipped with sensory, computing, operating and communication capabilities, which provides data, information and services to its local environment or to a cloud environment. Awareness of the real world and the dynamics of its integration within the manufacturing system is based on three basic principles: identification (Who am I?), Exploration of the service (What can I offer?) And active interconnection (Where are my collaborators?) [5].

Based on the objectives and characteristics defined above, the prototype of the CPPS demonstrator can ideally be composed of the following production modules:

- Smart warehouse module where the product components are managed and stored

- Assembly modules where different product subassemblies are manufactured.

- Processing/customization station. Here, customization operations such as engraving on the casings of the product are performed.

- Quality inspection and packaging station. Here the compliance of the product with the customer's order is verified. Furthermore, the product is packaged appropriately (e.g. modules, instructions for use, carton)

- Station for manual assembly where the products that cannot be fabricated automatically are manufactured by a human operator. The station also has the role, to complete an order, in case the automated production system fails.

- Training station. Here the human operator is trained how to operate and/or perform maintenance on different workstations and/or how to make different customizable products.

- Transport system. It ensures transportation between all the above production modules except the training station.

It is intended that the prototype of the adapted manufacturing system will have a modular and flexible construction, which can allow easy extension with other modules. For example, the modular construction allows a "line follower" transport system with precise dockings regardless of the topology chosen for the CPPS (Fig. 1).

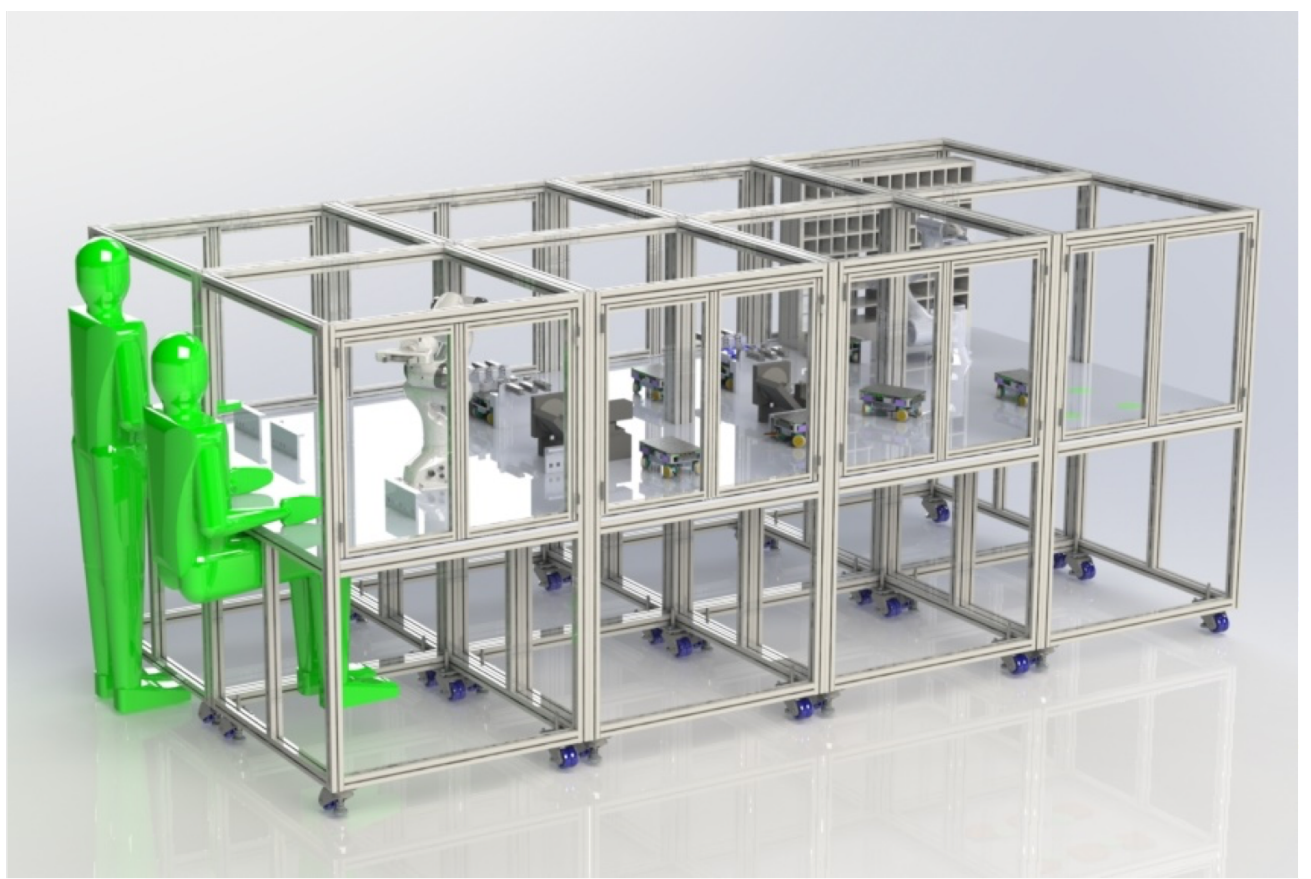

Figure 1 CPPS demonstrator concept 


\section{Conclusions}

Many years have passed since the early 20th century, when Henry Ford mastered the moving assembly line and started the age of mass production. Nowadays on the verge of a fourth industrial revolution, factories have changed dramatically. There is a constant unspoken contest between them, to see which one can achieve the lowest production times and deliver the best quality to their customers. In order to achieve these objectives, the companies must be up to date with what is being state of the art technologies today. Considering this, we proposed to identify the main requirements for building a CPPS demonstrator which is able to bring added value for the local industry but also serve as an academic research and teaching platform. Within this research we provided the foundation of building a such demonstrator by identifying the most important characteristics which a CPPS demonstrator has to accomplish.

\section{Acknowledgment}

This work is supported through the DiFiCIL project (contract no. 69/08.09.2016, ID P_37_771) co-founded by ERDF through the Competitiveness Operational Programme 2014-2020.

\section{References}

1. E. V. G. D. V. Z. D. Mourtzis, "Cyber- Physical Systems and Education 4.0-The Teaching Factory 4.0 Concept," Procedia Manufacturing, vol. 23, pp. 129-134, 2018.

2. " SmartFactory KL," [Online]. Available: https://smartfactory.de. [Accessed 2019].

3. A. P. J. P. Iris Gräßler, "Creation of a Learning Factory for Cyber Physical Production Systems," Procedia CIRP, vol. 54, pp. 107-112, 2016.

4. R. J. B. G. E. J. N. Zsolt Kemeny, "The MTA SZTAKI Smart Factory: platform for research and project-oriented skill development in higher education," Procedia CIRP, vol. 54, pp. 53-58, 2016.

5. Pirvu, B.-C.; Schlick, J.; Hodek, S.; Zühlke, D.: Conceptual overview of a smart-factory architecture. 6th International Conference on Manufacturing, Science and Education (MSE), Sibiu, Romania, June 2013 